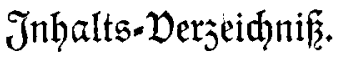

$\S 1$. Einleitung. Beltungăberei歹 ber Stäbteoro. 1

Seite

\section{Titel I.}

Bon ben Gruttolagen ber ítübtifđen Berfafiutut.

8. Umfang unb Beränderung beș Stabtbezirfa 2

\$3. IImfang ber Stabtgemeinde. Begriff ber

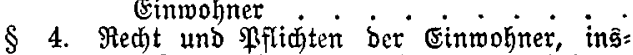
befondere bie (Betmeindeabgabenpfliogt . 3

$\S$ 5. Begriff und Erwerb bes Bürgerredts . 10

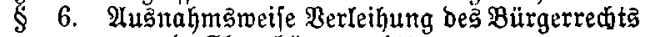
unb Eghentürgetrechts . . . . . 13

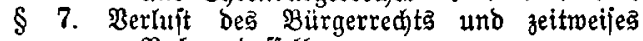
§uhen beffelben ......... 13

\$ 8. Begriff ber Eltabtgemeinde . . . . . 16

9. Bertretung ber Stabtgemeinbe. . . 16

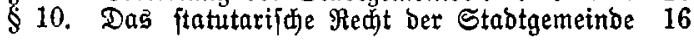

\section{Titel II.}

Bon ber Bujantmenietunn unt $\mathfrak{B a h \mathfrak { l }}$ ber Stantperardietenuerjammlung.

\$11. 3ujammenjęung det StabtverorbnetenverF. (3ahl bet Stabtoeroroneten) $\quad . \quad 17$

$\S 12$. Biloung ber $\mathfrak{B a h l a b t h e i l u n g e n ~} .: \cdots 18$ 
\$13. Billountg Der \$ablgexirfe . . . . . . 20

$\$ 14$. Bef́timmung bur 3afl ber Stabtverorontetent in ben aus mehreren Srtj巾aften be= itehenben Stabtgemeinben . . . 20

$\$ 15$. Bertretung ber 5ुaugbefiper in ber Stabt: perorbnetenverjammlung . . . . . 20

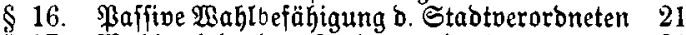

17. Wahlperiobe ber Stabtverorbneten ... 21

$\$ 18$ Führung uno seridtigung ber Bählerlifte 22

$\$$ 19. Dffenlegung $\mathfrak{u}$. Feftitellung b. Bählerlijtc. Berfahren für Einmentungen gegen bie Ridtigfeit ber Mählerlifte. . . .

$\$ 20$, 3eit ber Stabtweroronetemmahlen, ber regel=

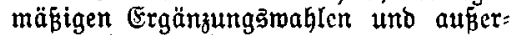
gewöhnliden Exfátwahlen . . . . 24

\$21. Ergänzung Der Bertreter ber S2auabefiłer 25

22. Berufung ber $\mathfrak{B a ̈ h l e r ~ z u ~ b e n ~} \mathfrak{B a h l e n}$. 25

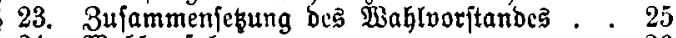

24. Mahlverfahren . . . 26

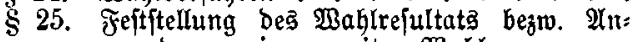
orbnung einer zreiten $\mathfrak{B a h l}$. . 26

\$26. Wahlprototole

$\$ 27$. Amtsoauer und Einfübrung der Stabtver: orbneten . . . . . . 28

\section{Titel III.}

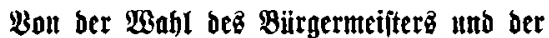
Brigearbneten (Magititratêperionen.)

\$28. 3ahl ber Beigeorbmeten . . . . . . 29

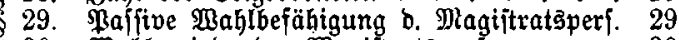

30. Mahlperiode Der Magijtrataperfonen, 30

31. פ̉ahlverfahren ...... . 31

32. Beftätigung ber Magiftratsperfonen . . 31

33. (Einführung unb Bereibtgung ber Mlagijtrats: perfonen. . . . . 33 
Titel IV.

Soite

\section{Bon Den Gefduaften Der Stadtverorducten= berjantmling.}

\$34. Eompetenz b. Etastverorbnetenverjammlung 33

35. Alusführung ber Stabtoeroronetenbejdlüfje 36

$\$ 36$. Borit ber Stabtoeroronetenverfammlung $\mathfrak{u}$.

Beidhlusifaffung berfelben . . . . . 36

\$37. 3eit ber 3ufammenberufung . . . . . 37

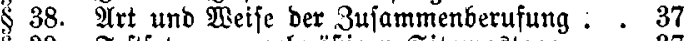

39. Zeftjegung regelmäß̈iger Sibungstage . . 37

\$4. Erforberniffe Der Befdlubfähigfeit o. Stabt= verorbnetenderjammlung . . . . . 37

\$ 41. Berfahren bei collibirenden Intereffen uno bei Prozeffen gegen Magiftratsperjonen 38

\$42. Seffentlidfett bor Situngen . . . . . 39

$\$ 43$. Seitung Der Berhandlungen burd ben Bor= fizenden u. IUfredterhaltung b. Sromung itt ben Sikungen . . . . . . . 39

\$ 44. Siłungäprotofolle und (5eí)äftsororonung. 39

$\$ 45$. Bejdlukfafiung über ba马 Bemeinde=Bürger: und Etiftungảs:3ermögen . . . 41

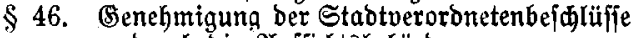
Duld bie 2Lffictatabeförbe . . . . 42

\$ 47. Berfahren bei Beräuß̧erung u. Berpadjung ftäbtijđer (Brunbitüđe . . . . 43

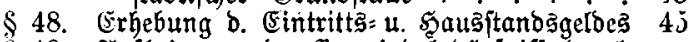

$\$$ 49. 2uffringung ber (Bemeinbebedürfniffe burd Steuern .46

$\$ 50$. Bephlüffe megen Seifitung von şano= und Spambienitent . . . . 49

\$51. Berwaltung Der Bemeinberwalburgent . 50

$\$ 52$. Wahl bes (Gemeindeeinnel)mers und geftint= nutng finter Ћ̆aution. . . . . 50 


\section{- VIII -}

\section{Titel V.}

Seite

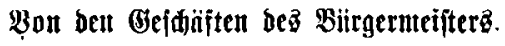

§53. (3eidäfte besิ Bürgermeifters . . . . . 51

54. Berwaltungsbeputationen u. Rommiffionen 55

55. Rezirfsuorjteher . . . . . . 56

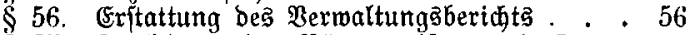

\$57. Junftionen bes Bürgermeifters, als Drgan ber Staatşgetwalt. . . . 57

\section{Sittel VI.}

Bon ben (Geljälterı uแb Penfiouen.

\$28. Feftetezung ber Befoldungen. . . . . . . 59

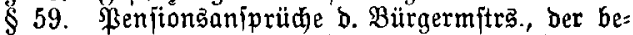
foldeten Beigeorbneten und ber befoldeten Gemeinbefeamten . . . . . . 60

\section{Tite! VII.}

\section{Bon bem Genteindehatảbalte.}

$\S 60$. Entmurf, Dffenlegung und Feftitellung beß Salishaltsetats . . . . . . 62

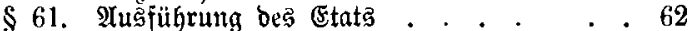

62. Beitreibung ber Bemeindeabgaben . . . 63

\$63. Revifion, Prüfung und Feftftellung ber Jahreäredgunng. Entlaftung Deș Ein= nehmers . . . . . . . . . . 63

$\S 64$. 3eit ber Feftitellung ber Эabresirechnung, Mittgeilung berf. an bie $\mathfrak{A}$ uffichtabegörbe. 63

$\S 65$. Führutg des \&agerbud 
Seite

\section{Titel VIII.}

Bou ber (Cituridtunty ber ftäbt. Werfafiung mit tollegialijidem Miagifitrat.

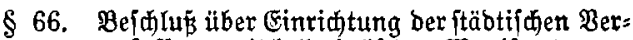
faffung mit follegialifidem Magiftrate . .

$\S 67$. Armenbung ber Borfhr. ber Titel I bis VII im Falle joldjer Einridtung . . . 65

$\S 68$. 3ujammenjeţung b. folfegialifhen Dagiftrats 65

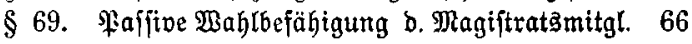

$\$ 70$. Mahlperiobe ber Magiftratsmitglieber $\quad 66$

$\$$ 71. Bejtätigung ber $\mathfrak{M a h l}$ berjelben . . . . 67

$\$ 72$. Walj $D$. Woriikenden b. Stabtveroroneten= verfammlung, Deffen Stellvertreters und bes Sd)riftführers. . . . . . . . 67

\$ 73. Mittheilung ber Stadtveroronetenbeidlüffe an Den Magiftrat. . . . . . . 68

$\$ 74$. Competenz Dę follegtalifoen Mtagiftrats.

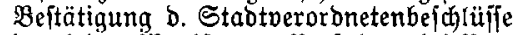

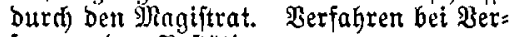
fagung ber Bejtätigung . . . . 68

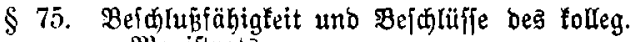
Mlagiftrats . . . . . . 70

§ 76. Beforgung ber Beffäfte bes Maạiftrats Durd ben Bürgermeifter in eiligen Făllen. 71

$\S 77$. Berwaltungs̄beputationen $\mathfrak{u}$. Siommiffionen 71

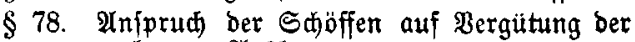
baaren 2 uslagen ....... 72 


\section{$-\mathrm{X}-$}

Titel IX.

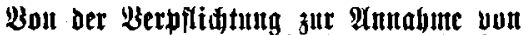

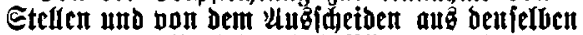

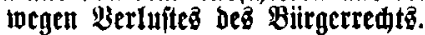

$\S 79$. Berpflidtung jur $\mathfrak{A} n$ nafme unbefoldeter fläbtif́ner 2lemter. . . . . . . 72

$\S 80$. Berluft ber 2emter unb Suspenfton im Falle bes ruhenben Bürgerredto .

Titel $\mathbf{X}$.

Bon Der Dberaufifint liber die Stabtuerwaltung.

\$81. Auffichtąbehörben . . . . . . . . . 75

$\$ 82$. Bejhmerbe in Bemeindeangelegenheiten. Frifít ber Befdiwerben. . . . . . 76

\$ 83. Bejdlitfie b. Stabtwerorbneten, weldje beren Befugnifje überjareiten unb bie Gejeşe verlesen ..........

\$ 84. 3wangอ̨meife Eintragung gejeţr. Beiftungen in ben syaushaltsetat ..... 78

$\$$ 85. Frift bes ßecurjes . . . . . . . . 79

$\$ 86$. 2luflöfung b. Stabtveroronetenverfammlung 79

$\$ 87$. Dienitwergchen ber Bemeinbebeamten . . 80

\section{TiteI XI.}

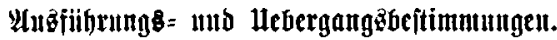

\$ 88. Musfübrung ber Stäbte=Dronung . . 82

89. Miederheritellung bes Eenius . . . 82

$\$ 90$. Einführung ber Stäbteoronung in b. nidit im Bürgermeiftereiverbanbe mit anderen Bemeinden befindlidien Stäbten . . 82

\$ 91. Einfühtrung ber Stäbtcorbmung in ben mit anberen Bemeinben im Bürgermeifterei: verbanbe befindliden Stäbten ... S 
\$92. Belaffung ber Gemeinbebeamten in ihren 2Yemtern und Einfünften . . . . 83

$\$ 93$. Einführung $\delta$. Stäbteoronung in benjenigen Stäbten, in weldhen bie Bemeinbeorong. vom 23 . כuli 1845 nod in Mitffamfeit fid befindet . . . . . . . . . . 83

\$94. Regulirung b. Bergältniffe ber vormals unt mittelbaren beutiden Reidştände und Der Befitzer von Etanbeşerrlidfeiten . 84 
